\title{
Frequency up-converted radiation from a cavity moving in vacuum
}

\author{
A. Lambrecht ${ }^{a}$, M.T. Jaekel ${ }^{b}$ and S. Reynaud ${ }^{a}$ \\ ${ }^{a}$ Laboratoire Kastler Brossel*, Université Pierre et Marie Curie, case 74, 4 place Jussieu, 75252 Paris, France \\ ${ }^{b}$ Laboratoire de Physique Théorique de l'Ecole Normale Supérieure ${ }^{\dagger}, 24$ rue Lhomond, 75231 Paris, France
}

(to appear in European Physical Journal D3, 1998)

We calculate the photon emission of a high finesse cavity moving in vacuum. The cavity is treated as an open system. The field initially in the vacuum state accumulates a dephasing depending on the mirrors motion when bouncing back and forth inside the cavity. The dephasing is not linearized in our calculation, so that qualitatively new effects like pulse shaping in the time domain and frequency up-conversion in the spectrum are found. Furthermore we predict the existence of a threshold above which the system should show self-sustained oscillations.

PACS: $42.50 . \mathrm{Lc}-03.70 .+\mathrm{k}-12.20 .-\mathrm{m}$

Vacuum field fluctuations exert radiation pressure on scatterers in vacuum. For a pair of mirrors at rest this effect is well known as Casimir effect [1]. When a mirror is moving radiation pressure of vacuum fluctuations leads to a dissipative force which opposes itself to the mirrors motion. This force is known to arise as soon as the mirror has a non-uniform acceleration [2]. Accordingly the electromagnetic field remains not in the vacuum state but photons are emitted by the scatterer into vacuum [3]. Radiation from a moving mirror and the associated radiation reaction force imply that dissipative effects are associated with the motion of mirrors in vacuum, although this motion has no further reference than vacuum itself. Since these effects challenge the principle of relativity of motion in vacuum, it would be very important to obtain experimental evidence for them and to study their characteristics in detail.

Motion-induced radiation can be interpreted as a result of dephasing of vacuum fields depending on the mirrors motion. The order of magnitude of the dephasing is expected to be the ratio between the mirror's velocity $v$ and the speed of light $c$. For most conceivable motion of a macroscopic object, the velocity $v$ cannot greatly exceed the sound velocity and is thereby much slower than that of light. This is why motion-induced radiation is very small for a single mirror oscillating in vacuum. This conclusion holds for perfectly reflecting mirrors as well as for partly transmitting ones.

A number of works have been devoted to photon production inside the cavity built with a pair of perfectly reflecting mirrors moving in vacuum 4 . 7. However no

\footnotetext{
*Laboratoire de l'Ecole Normale Supérieure et de l'Université Pierre et Marie Curie associé au CNRS

${ }^{\dagger}$ Laboratoire du CNRS associé à l'Ecole Normale Supérieure et à l'Université Paris Sud
}

predictions can be made for the amount of radiation emitted outside the cavity when the resonator is treated as a closed system. In contrast the resonant enhancement is found to be determined by the cavity finesse when the cavity is treated as an open system [8] from which the photons can escape. Motion induced radiation, that is photon emission outside a cavity oscillating in vacuum is resonantly enhanced by the cavity finesse when compared to the radiation from a single oscillating mirror. The resonant enhancement occurs when the mechanical frequency is a multiple of the lowest cavity mode. Even and odd multiples correspond respectively to breathing modes, where the mechanical cavity length changes periodically, and to translation modes, where the cavity moves as a whole [9]. The latter effect reminds radiation from a single mirror inasmuch as vacuum fluctuations are the only reference for the cavity motion. However the order of magnitude of photon emission may greatly exceed the one from a single mirror. From an experimental point of view the cavity is so far the most interesting system to look for an experimental observation of dissipative effects of vacuum fluctuations.

Inside a cavity the field undergoes many reflections before leaving the cavity through one of the mirrors. The number of round-trips of the field is roughly given by the cavity finesse. In loose terms, one may define an effective velocity where the physical velocity $v$ normalized by the speed of light is multiplied by the number of round-trips inside the cavity. Effective velocity and thus motioninduced radiation become the larger the higher is the cavity finesse. The effective velocity is no longer a material velocity and may therefore approach the speed of light. In contrast to the single mirror's case, qualitatively new effects are expected, such as the formation of a pulse bouncing back and forth in the cavity [6,7]. Since the pulse duration is shorter than the time of flight through the cavity, the radiation spectrum should also contain various frequencies corresponding to higher-order cavity modes and thus exceeding the mechanical frequency.

These effects cannot be obtained with a linear treatment as the one used in [9]. In such a treatment the field scattering is supposed to be linear in the mirrors motion. The field-mirror interaction corresponds to a coupling of the vacuum field radiation pressure, which is quadratic in the field, to the mirrors mechanical motion. Photons are thus emitted in pairs. In the linear regime the generation of motion-induced radiation is analogous to a parametric process during which the mechanical excitation is transformed into a pair of photons. Due to energy conserva- 
tion the sum of their frequencies equals the oscillation frequency. Therefore motion-induced photons are only emitted at frequencies smaller than the excitation frequency. The linear assumption is correct as long as the total field dephasing due to interaction with the moving reflector remains small. The field dephasing from one reflection scales with the mirrors velocity over the speed of light. The linear assumption is always satisfied for a single macroscopic mirror.

However for a cavity the crucial parameter is the effective velocity and the total field dephasing can become important for a high finesse cavity. As a consequence we expect frequency multiplication to occur which generates frequencies larger than the mechanical excitation frequency. The linear treatment, which predicts the emission frequencies to be smaller than the oscillation frequency, then looses its validity and has to be replaced by a treatment which fully accounts for a large field dephasing produced through successive reflections of the field onto the mirrors. This treatment will be called non-linear in the following although the scattering is still linear in the field.

The aim of the present paper is to give a treatment of the radiation emitted by a cavity moving in vacuum which takes into account both the effect of accumulated field dephasing and the open character of the cavity. In particular we will study the explicit dependence of motional radiation on the two experimentally accessible parameters, the mirrors velocity and the cavity finesse. We will first introduce general calculation techniques for a single mirror and for a cavity moving in vacuum with an arbitrary motion. We will then concentrate on a particular motion, the harmonic oscillation of the mirrors, which allows to evaluate in closed analytical form the correlation functions of the radiation through a special parametrization of the motion. We will give expressions for the time-dependent radiated energy as well as the frequency-dependent radiation spectrum.

\section{SINGLE MIRROR MOVING IN VACUUM}

Neglecting all effects related to polarizations, the electromagnetic field $\Phi$ is considered as a scalar function of one space variable, $t$ and $x$, and will be written as a sum of two counterpropagating components $\varphi$ and $\psi$ which are functions of two light-cone variables $u$ and $v$

$$
\begin{array}{rl}
\Phi(t, x) & =\varphi(u)+\psi(v) \\
u=t-x & v=t+x
\end{array}
$$

For the sake of simplicity, the velocity of light is set to unity. We limit ourselves here to two-dimensional spacetime calculations. As is well known from the analysis of squeezing experiments [10], the transverse structure of the cavity modes does not change appreciably the results obtained from this simplified model. Each transverse mode is correctly described by a two-dimensional model as soon as the size of the mirrors is larger than the spot size associated with the mode. The two-dimensional model thus corresponds to a situation where one transverse mode is efficiently coupled to the moving mirrors.

We now represent the mirror's motion either by a function $x=q(t)$ associated with the trajectory or as a monotonous function $v=V(u)$ relating the light cone variables $u$ and $v$ of the light rays intersecting on the mirror's trajectory. A propagation component $\varphi_{\text {out }}$ of the output field can then be written as a function of the input fields $\varphi_{\text {in }}$ and $\psi_{\text {in }}$ and of the function $V$

$$
\begin{aligned}
\varphi_{\text {out }} & =\sqrt{R} \psi_{\text {in }} \circ V+\sqrt{T} \varphi_{\text {in }} \\
T & =1-R \\
(h \circ g)(u) & =h(g(u))
\end{aligned}
$$

The symbol $\circ$ represents the composition law for functions. The coefficients $\sqrt{R}$ and $\sqrt{T}$ are the reflection and transmission amplitudes describing scattering upon the mirror. For the sake of simplicity, we have assumed these coefficients to be real and frequency independent.

We now recall the standard calculation of the energy radiated into vacuum by the moving mirror [2]. The input fields are supposed to be in the vacuum state and characterized by the correlation function

$$
\left\langle\varphi_{\mathrm{in}}(u) \varphi_{\mathrm{in}}(\bar{u})\right\rangle=-\frac{\hbar}{4 \pi} \ln |u-\bar{u}|-\frac{i \hbar}{8} \varepsilon(u-\bar{u})
$$

The first term corresponds to the anticommutator and is state-dependent whereas the second term gives the commutator contribution and is state-independent. Since $\varepsilon$ is the sign function, it is clear that the field commutator remains unchanged under the transformation (2) where $u$ is replaced by a monotonous function $V(u)$. The change of the correlation function between the input and output field is given by the following function which depends on the field anticommutators only

$$
\begin{aligned}
C(u, \bar{u}) & =\left\langle\varphi_{\text {out }}^{\prime}(u) \varphi_{\text {out }}^{\prime}(\bar{u})\right\rangle-\left\langle\varphi_{\text {in }}^{\prime}(u) \varphi_{\text {in }}^{\prime}(\bar{u})\right\rangle \\
& =-\frac{\hbar R}{4 \pi}\left(\frac{V^{\prime}(u) V^{\prime}(\bar{u})}{(V(u)-V(\bar{u}))^{2}}-\frac{1}{(u-\bar{u})^{2}}\right)
\end{aligned}
$$

Throughout the paper, the prime signifies a derivative of a function with respect to its argument. The energy density $e_{u}(u)$ radiated per unit time is given by the function $C(u, \bar{u}=u)$ evaluated at coinciding points through a point splitting regularization procedure [2]

$$
\begin{aligned}
e_{u}(u) & =C(u, u)=-\frac{\hbar R}{24 \pi} \mathcal{S} V(u) \\
\mathcal{S} V & =\frac{V^{\prime \prime \prime}}{V^{\prime}}-\frac{3}{2}\left(\frac{V^{\prime \prime}}{V^{\prime}}\right)^{2}
\end{aligned}
$$

The function $\mathcal{S} V$ is the Schwarzian derivative of $V$. No radiation is emitted when the reflector has a uniform acceleration, which corresponds to a vanishing Schwarzian derivative $\mathcal{S} V$. The total energy radiated by the moving 
mirror can then be obtained by integrating the energy density over $u$. In the following, we will concentrate on the particular case of an oscillatory motion of mechanical frequency $\Omega$. In this case the energy $E_{u}$ radiated per period is read as

$$
E_{u}=\int_{0}^{\frac{2 \pi}{\Omega}} e_{u}(u) \mathrm{d} u
$$

In order to characterize the radiation we have also to describe its spectral properties. The radiation spectrum may be represented as a density of photons obtained from the Fourier transform of the two point function $C(u, \bar{u})$. We will turn to its description later on.

\section{CAVITY MOVING IN VACUUM}

The vacuum field is defined on both sides of the cavity as in the previous section. The relation between input and output fields is a generalization of (2) which corresponds to the standard Fabry-Perot theory

$$
\begin{aligned}
\varphi_{\text {out }} & =-\sqrt{R_{2}} \psi_{\text {in }} \circ V_{-1}+\sqrt{R_{1}} T_{2} \sum_{n \geq 0} r^{n} \psi_{\text {in }} \circ V_{n} \\
& +\sqrt{T_{1}} \sqrt{T_{2}} \sum_{n \geq 0} r^{n} \varphi_{\text {in }} \circ U_{n} \\
T_{1} & =1-R_{1} \quad T_{2}=1-R_{2} \\
r & =\sqrt{R_{1}} \sqrt{R_{2}}=e^{-2 \rho}
\end{aligned}
$$

The reflection and transmission amplitudes of the two mirrors are related through unitarity conditions. The coefficient $r$ determines the attenuation factor of the field on a single cavity round-trip. It can also be written as a function of the cavity losses $\rho$. Throughout the paper we will use $\rho$ when we consider the experimentally interesting case of a high finesse cavity with $\rho \ll 1$. In the more general case the reflection coefficient $r$ will be used. The functions $U_{n}$ and $V_{n}$ represent the light cone variables associated with the various input rays which are transformed into the output light ray $u$ by the cavity. When the cavity is at rest they are given by simple relations

$$
\begin{aligned}
& U_{n}(u)=u-2 n L \\
& V_{n}(u)=u-(2 n+1) L
\end{aligned}
$$

The length $L$ of the cavity is measured as a time of flight between the two mirrors; the two mirrors are supposed to be located at $x= \pm L / 2$ respectively; the ray $V_{-1}$ represents the particular case where the field has been directly reflected back by the first encountered mirror without entering the cavity.

We may deduce the field emitted by the vibrating cavity through the same expression (7) as for the motionless cavity, but with functions $U_{n}$ and $V_{n}$ now given by the procedure sketched on figure 11.

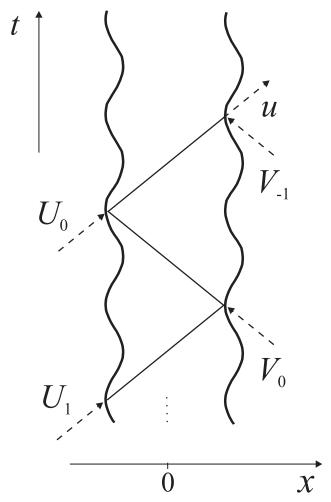

FIG. 1. Space-time diagram of on arbitrary field trajectory bouncing back and forth inside the cavity. Both mirrors are supposed to follow a harmonic motion. Light rays are indicated by null lines, i.e. straight lines making a $45^{\circ}$-angle with the time and space axis.

The functions $U_{n}$ and $V_{n}$ corresponding to the various light rays in figure 1 are built up through a functional iteration,

$$
\begin{aligned}
U_{n}=f_{2 n} & V_{n}=f_{2 n+1} \\
f_{-1}=g & f_{0}=I \\
f_{2 n}=g^{-1} \circ f_{2 n-1} & f_{2 n+1}=h \circ f_{2 n}
\end{aligned}
$$

where $I$ is the identity function $(I(u)=u)$, while the two functions $h$ and $g^{-1}$ (inverse of $g$ ) represent the trajectories of the mirrors

$$
\begin{aligned}
& x=q_{1}(t) \Longrightarrow v=h(u) \\
& x=q_{2}(t) \Longrightarrow v=g(u) \Rightarrow u=g^{-1}(v)
\end{aligned}
$$

The function $f_{2 n}$ results from $n$ successive compositions of the function $g^{-1} \circ h$. This construction is quite analogous to the one described in [7], but it is written here such that it may be applied to an open cavity.

In a linear treatment [9] the total field dephasing remains small. At every reflection the field acquires a dephasing due to the mirrors motion $2 q_{i}(t),(i=1,2)$. In this case the composition of functions (9) is reduced to the summation of the mirrors motion. The accumulated dephasing after $M$ roundtrips inside the cavity is now seen to be simply $M$ times the dephasing due to a single roundtrip. The scattered field then has a temporal variation which reproduces the mirrors motion. With these approximations the results of the linear treatment are recovered.

However, if the field undergoes a great number of roundtrips inside the cavity, the total dephasing does not remain small and a linearization is not valid anymore. The dephasing has then to be calculated through the general composition law (9). Following the same procedure as for the single mirror, we calculate the density of energy $e_{u}(u)$ radiated to the right through the twopoint correlation function defined as in (4) by letting $\bar{u}$ come to coincidence with $u$. As all functions now depend 
on a single parameter $u$, we omit this parameter in the expression for the energy density

$$
\begin{aligned}
e_{u}=- & \frac{\hbar}{4 \pi}\left\{\frac{R_{2}}{6} \mathcal{S} f_{-1}-2 T_{2} \sum_{n \geq 0} r^{n+1} \frac{f_{-1}^{\prime} f_{2 n+1}^{\prime}}{\left(f_{-1}-f_{2 n+1}\right)^{2}}\right. \\
& +\frac{T_{2}^{2} R_{1}}{6} \sum_{n \geq 0} r^{2 n} \mathcal{S} f_{2 n+1}+\frac{T_{1} T_{2}}{6} \sum_{n \geq 0} r^{2 n} \mathcal{S} f_{2 n} \\
& +T_{1} T_{2} \sum_{n \neq m \geq 0} r^{n+m} \frac{f_{2 n}^{\prime} f_{2 m}^{\prime}}{\left(f_{2 n}-f_{2 m}\right)^{2}} \\
& \left.+T_{2}^{2} R_{1} \sum_{n \neq m \geq 0} r^{n+m} \frac{f_{2 n+1}^{\prime} f_{2 m+1}^{\prime}}{\left(f_{2 n+1}-f_{2 m+1}\right)^{2}}\right\}
\end{aligned}
$$

Compared to the radiated energy density of a single mirror (5), we now find a sum of Schwarzian derivatives corresponding to different numbers $n$ of round-trips inside the cavity, as well as new terms arising from the interference between light rays having undergone a different number of roundtrips.

The derivatives appearing in the upper equation are iteratively deduced from each other through relations (9) and the chain rules associated with derivation of composed functions

$$
\begin{aligned}
(g \circ h)^{\prime} & =h^{\prime} g^{\prime} \circ h \\
\mathcal{S}(g \circ h) & =\mathcal{S} h+h^{\prime 2}(\mathcal{S} g) \circ h
\end{aligned}
$$

In the general case of arbitrary motions of the two mirrors, the various relations which have been written in the present section allow to compute the energy density radiated by a cavity built with partly transmitting mirrors.

\section{HARMONIC MOTIONS AND PERIODIC ORBITS}

From now on we focus our attention on configurations which have been shown to be the most efficient ones to generate motion-induced radiation [9] and which furthermore allow us to put the problem in a simpler form.

We consider that the two mirrors follow harmonic motions at such a frequency that the motion-induced effects, i.e. motional radiation and motional force, are resonantly enhanced by the multiple interference taking place inside the cavity. The frequency $\Omega$ of the harmonic motion is thus supposed to be such that $\Omega L$ is a multiple of $\pi$

$$
\Omega L=K \pi
$$

The amplitudes of the two motions are supposed to have the same absolute value with either opposite or identical signs depending on the parity of the integer number $K$

$$
\Omega q_{1}(t)=-\frac{K \pi}{2}-\beta \sin \left(\Omega t-\frac{(K+1) \pi}{2}\right)
$$

$$
\begin{aligned}
\Omega q_{2}(t) & =\frac{K \pi}{2}-\beta \sin \left(\Omega t+\frac{(K+1) \pi}{2}\right) \\
\beta & =\operatorname{th}(\alpha)
\end{aligned}
$$

We have written the two equations of motion in terms of dimensionless numbers. In particular, one distinguishes two parameters $\alpha$ and $\beta$. $\beta$ represents the ratio between the maximal velocity of the mirrors and the velocity of light while $\alpha$ plays the role of a rapidity which will be found to add up through successive reflections when one considers the composed motion of both mirrors.

When $K$ is an even number, the upper equations describe a situation where the two mirrors are oscillating such that the length of the cavity changes periodically. In the opposite case, the cavity performs a global oscillation with its length kept constant. The two cases will be called even and odd modes respectively in the following. A cavity motion corresponding to an odd mode is reminiscent of motion-induced radiation from a single oscillating mirror, but in addition here the radiation is resonantly enhanced inside the cavity. These statements follow from the linearized approach [9] but we expect a similar behavior to take place in the full treatment developed in the present paper.

There exist periodic orbits such that the optical length seen by the field bouncing back and forth in the cavity is the same on successive round-trips despite the motion of the mirrors [7]. These orbits correspond to particular values $\tilde{u}$ of the light-cone variable $u$ such that the iteration procedure leads to expressions similar to the ones obtained when the mirrors are at rest (cf. (8)). Although definition (9) of $f_{p}$ is different depending on whether $p$ is even or odd, the periodic orbits generalize the usual resonance condition of the Fabry-Pérot theory

$$
f_{p}(\tilde{u})=\tilde{u}-p L
$$

They therefore give rise to a constructive interference effect, analogous to that occurring for a motionless cavity. Since the light rays corresponding to a periodic orbit encounter the mirrors at the same position after an arbitrary number of round-trips, the composition (9) of motions leads to a simple power law for the derivatives evaluated after $n$ roundtrips as well as for the Schwarzian derivatives

$$
\begin{aligned}
f_{p}^{\prime}(\tilde{u}) & =e^{2 p \alpha} \\
\mathcal{S} f_{p}(\tilde{u}) & =\mathcal{S} f(\tilde{u}) \frac{1-e^{4 p \alpha}}{1-e^{4 \alpha}}
\end{aligned}
$$

There exist two sets of periodic orbits which correspond to opposite values of $\alpha$ in (16). These two periodic orbits attract (respectively repel) the neighboring trajectories, when $e^{4 \alpha}$ is greater (respectively smaller) than unity. Only the attractive orbit is expected to give rise to a large enhancement of the motional radiation.

In expression (11) of the energy density the Schwarzian derivative (16) is multiplied by the squared reflection 
coefficient after $n$ roundtrips $r^{2 n}$. Summation over the number of roundtrips leads then to a geometric progression of $r^{2 n} e^{8 n \alpha}$. The first factor represents the attenuation of the energy density associated with the cavity losses through the two mirrors. The second one accounts for the parametric amplification of the field associated with the mirrors' motion. As a consequence, the energy density takes large values when the parametric amplification compensates the losses. In fact, a divergence of the energy density should occur when $r e^{4 \alpha}$ approaches unity. This corresponds exactly to the oscillation threshold of a mechanically excited parametric amplifier. Let us notice that the approach developed in the present paper does not remain valid above this threshold.

We have focussed our attention here on the case where periodic orbits correspond to light rays meeting the two mirrors at their mean positions, respectively $-\frac{L}{2}$ and $\frac{L}{2}$. There exist more general situations where the light rays meet the mirrors at other positions [7] which will not be considered here. Notice that the particular case studied in the present paper is interesting from an experimental point of view since it corresponds to the maximum value of the parameter $\alpha$ for motions having a given frequency and a given amplitude.

In the following, we will restrict our attention to the cases of practical interest where the physical velocity of the mirror remains small when compared to the velocity of light. As discussed in the Introduction, this condition is always met for macroscopic mirrors. It implies that a single reflection produces a small dephasing on the field and, thereby, small radiation effects. Precisely, this means that the quantity $\mathcal{S} f$ which appears in (16) has an extremely small value while the factor $e^{2 \alpha}$ is very close to unity. It follows that a large number $n$ of roundtrips is needed to obtain a factor $e^{2 n \alpha}$ differing appreciably from unity and therefore giving rise to a noticeable radiation. We show now that this assumption permits to perform the functional iteration in an analytical manner.

The crucial point is that the functional iteration (9) may in this case be restricted to the sub-space of periodic functions $h$ corresponding to homographic relations between the phases $e^{i \Omega u}$ and $e^{i \Omega h(u)}$

$$
e^{i \Omega h(u)}=\frac{a e^{i \Omega u}+b}{b^{*} e^{i \Omega u}+a^{*}} \Longrightarrow A(h)=\left(\begin{array}{ll}
a & b \\
b^{*} & a^{*}
\end{array}\right)
$$

$a$ and $b$ are two complex constants and $a^{*}$ and $b^{*}$ their complex conjugates which can be gathered in a matrix $A(h)$ associated with the function $h$. Attention may be restricted to matrices having a determinant equal to unity. In the sub-space of functions (17), the composition of two functions merely corresponds to a product of their corresponding matrices.

$$
A(h \circ g)=A(h) A(g)
$$

Rigorously speaking, the function (17) does not correspond to sinusoidal trajectories (14) but rather to specific trajectories already considered by Law for dealing with photon production inside a closed cavity [6]

$$
\begin{gathered}
a=e^{i \phi_{a}} \operatorname{ch} \alpha=e^{i \phi_{b}} \operatorname{sh} \alpha \\
\sin \left(\Omega q-\phi_{a}\right)=-\beta \sin \left(\Omega t-\phi_{b}\right)
\end{gathered}
$$

where the reduced velocity $\beta$ gives the mirrors velocity compared to the speed of light. Variations of the phase factors $\phi_{a}$ and $\phi_{b}$ amount to displacements of the trajectory along the space and time axis. At the limit of small velocities however, the trajectory (19) is reduced to an ordinary sinusoidal motion (14)

$$
\beta \ll 1 \Longrightarrow \Omega q=\phi_{a}-\beta \sin \left(\Omega t-\phi_{b}\right)
$$

The difference between the two motions scales as the cube $\beta^{3}$ of velocity and is therefore extremely small for realistic motions of macroscopic mirrors. The effect of the trajectory (19) is thus indistinguishable from the effect of the sinusoidal motion for a single reflection.

If one considers two oscillating mirrors, the two functions $h$ and $g$, corresponding to the first and second mirror, are associated with two matrices $A(h)$ and $A(g)$ respectively. The matrix components are chosen to fit equations (14) of motion of the two mirrors

$$
\begin{aligned}
& A(h)=\left(\begin{array}{ll}
(-i)^{K} \operatorname{ch} \alpha & i^{K+1} \operatorname{sh} \alpha \\
(-i)^{K+1} \operatorname{sh} \alpha & i^{K} \operatorname{ch} \alpha
\end{array}\right) \\
& A(g)=\left(\begin{array}{ll}
i^{K} \operatorname{ch} \alpha & (-i)^{K+1} \operatorname{sh} \alpha \\
i^{K+1} \operatorname{sh} \alpha & (-i)^{K} \operatorname{ch} \alpha
\end{array}\right)
\end{aligned}
$$

As the composition of the two functions corresponds to a product of their matrices, the composition law naturally produces a homographic function when the number of reflections becomes large. This essential feature will be used in the following to compute the temporal and spectral characteristics of motional radiation from purely algebraic manipulations of the associated matrices. In particular, the functions $f_{p}$ in equation (9) corresponding to successive reflections of the field inside the cavity are obtained through matrix multiplication

$$
A_{p}=\left(\begin{array}{ll}
(-i)^{K p} \operatorname{ch} p \alpha & i^{2 K+1}(-i)^{K p} \operatorname{sh} p \alpha \\
(-i)^{2 K+1} i^{K p} \operatorname{sh} p \alpha & i^{K p} \operatorname{ch} p \alpha
\end{array}\right)
$$

The discrepancy between the composed functions built on the two motions (14) and (19) does not affect the results if $\beta \ll 1$. More precisely, the difference between the composed functions $f_{p}$ built on the two motions (14) and (19) remains of the order of $\beta^{2}$ when the number of iterations increases.

\section{PULSE SHAPING}

In the following, we will analyze the case of a single mirror following a trajectory (17) with an arbitrary velocity parameter $\alpha$. Although the hypothesis of a large 
rapidity $\alpha$ is not realistic for a single mirror, it can be used as a model for the composition of a large number of round trips inside the cavity. We will then come to the full treatment of the cavity where interferences have also to be accounted for.

The derivative $h^{\prime}(u)$ may be written from (17) as

$$
h^{\prime}(u)=\frac{1}{\left|a+b e^{-i \Omega u}\right|^{2}}
$$

$h^{\prime}(u)$ oscillates between the extremal values $\exp ( \pm 2 \alpha)$ which correspond to physical velocities of the mirror $\pm \beta$. For homographic functions (17), the Schwarzian derivative has the simple form

$$
\mathcal{S} h=\frac{\Omega^{2}}{2}\left(1-h^{\prime 2}\right)
$$

The total energy radiated to the right by a single mirror is then obtained by averaging the energy density (5) over one oscillation period

$$
E_{u}=\frac{\hbar R \Omega}{12} \operatorname{sh}^{2} \alpha
$$

This energy does not saturate when the parameter $\alpha$ increases although the velocity scales as th $\alpha$ and remains smaller than the velocity of light. The radiated energy is always greater than the squared velocity $\operatorname{th}^{2} \alpha$ which was the value suggested by the linear treatment [9]. However it is impossible to obtain appreciable radiation with a single oscillating mirror for velocities small compared to the speed of light. In the realistic case of a mirror moving at a small velocity the radiated energy as well as the number of emitted photons scale with $\alpha^{2}$.

We come now to the energy density radiated by an oscillating cavity, assuming that a large number of roundtrips is necessary to compensate the small velocity of the mirrors and thus get a noticeable radiation. The energy density $e_{u}$ may be obtained from (11) by using the following properties of the Schwarzian derivative of $f_{p}$ and of the first-order derivative $f_{p}^{\prime}$ respectively

$$
\begin{aligned}
\mathcal{S} f_{p} & =\frac{\Omega^{2}}{2}\left(1-f_{p}^{\prime 2}\right) \\
f_{p}^{\prime} & =\frac{1}{\operatorname{ch} 2 p \alpha+(-1)^{K} \operatorname{sh} 2 p \alpha \sin \Omega u}
\end{aligned}
$$

To plot the energy density for different linear and nonlinear regimes we introduce effective quantities

$$
\begin{aligned}
& \alpha_{\mathrm{eff}}=2 \alpha / \rho \\
& \beta_{\mathrm{eff}}=\operatorname{th}\left(\alpha_{\mathrm{eff}}\right)
\end{aligned}
$$

The effective rapidity $\alpha_{\text {eff }}$ is given by the roundtrip value of the rapidity multiplied by the cavity finesse $\rho^{-1}$. In contrast to the mechanical velocity $v$ normalized by the speed of light, which has to remain much smaller than 1 in any physical situation, the corresponding effective velocity $\beta_{\text {eff }}$ can become an important fraction of the speed of light when the field undergoes a large number of reflections inside the cavity. The maximal value of the effective reduced velocity is limited to $\beta_{\text {eff }} \sim 0.76$ by the divergence of the energy density at $\alpha_{\text {eff }}=1$. This value corresponds indeed to the threshold $r e^{4 \alpha}=1$ which has already been mentioned previously for the periodic orbits.

The variation of the energy density for different parameters $\alpha_{\text {eff }}$ is presented on figure 2. In the linear regime where $\alpha_{\text {eff }} \ll 1$ the temporal variation of the emitted energy is sinusoidal. When $\alpha_{\text {eff }}$ increases the energy concentrates in pulses which are periodically emitted by the cavity. This pulse shaping becomes the more pronounced, the width of the pulses the smaller, the larger becomes the effective rapidity and thus the accumulated field dephasing.

In the same manner as for the single mirror the total energy radiated by the cavity is computed by averaging the energy density over one oscillation period $2 \pi / \Omega$.

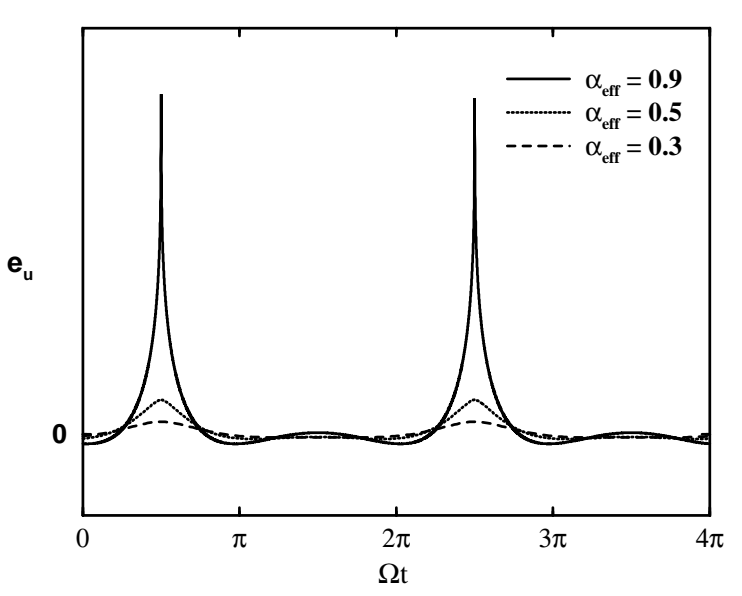

FIG. 2. Energy density emitted to the right by the cavity as a function of time for different effective rapidities $\alpha_{\text {eff }}$ and $r=0.99$. The top line of the frame corresponds to $10^{-3} \hbar \Omega^{2}$. With increasing values of the effective rapidity the energy starts to concentrate in pulses emitted periodically by the cavity. The pulses become very sharp close to the threshold of oscillation $\alpha_{\text {eff }}=1$.

As previously we will restrict our attention to a realistic case, where the mirrors velocity is small which justifies the use of the homographic relations. In this case we may replace the differences of functions in the denominators of (11) by their motionless values (8). Higher order contributions decrease with the inverse of their squared order. The sums over the number of roundtrips can then be performed and we find the following expression for the energy radiated to the right

$$
E_{u}=\frac{\hbar \Omega R_{2}}{12} \operatorname{sh}^{2} \alpha+\frac{\hbar \Omega T_{2}}{48}\left(\zeta_{u}(\alpha)+\zeta_{u}(-\alpha)-2\right)
$$




$$
\begin{aligned}
& -\frac{\hbar \Omega T_{2}}{8 \pi^{2} K^{2}}\left(\xi(\alpha)\left(\zeta_{u}(\alpha)-e^{-2 \alpha}\right)+\xi(-\alpha)\left(\zeta_{u}(-\alpha)-e^{2 \alpha}\right)\right) \\
\zeta_{u}(\alpha) & =\frac{\left(1-e^{-4 \rho}\right) e^{2 \alpha}+T_{1}\left(1-e^{2 \alpha}\right)}{1-e^{4(\alpha-\rho)}} \\
\xi(\alpha) & =\sum_{l=1}^{\infty} \frac{e^{2 l(\alpha-\rho)}}{l^{2} \operatorname{ch} 2 \alpha l}
\end{aligned}
$$

The energy $E_{v}$ radiated to the left can be obtained from the above formula by interchanging the indices 1 and 2 of the reflection and transmission coefficients. The total energy radiated in both directions is then evaluated as the sum of the two contributions

$$
E=E_{u}+E_{v}
$$

The total radiated energy would diverge for $\alpha=\rho$. However this limit is not reached as the energy density already diverges when $2 \alpha / \rho$ approaches unity. The reason for this difference is simply that the widths of the various contributions to the energy density decrease when the number of roundtrips $n$ increases, so that the contribution to the integrated energy increases less rapidly than the peak value of the energy density. As a consequence, the divergence of the peak density occurs before the divergence of the integrated energy. We remind here that our calculation does not remain valid above the oscillation threshold. Still it may be expected that a large amount of radiation is emitted above threshold.

For experimental reasons one might also be interested in the amount of energy stored inside the cavity. Let us first remark that inside the cavity the fields propagating to the right and to the left are not independent from each other due to the boundary conditions. All intracavity quantities thus contain the field components of both directions and are obtained as the sum of the two contributions. Having this argument in mind the intracavity energy, integrated over the cavity length $L=K \pi / \Omega$, is then with the same notations as above found to be

$$
\begin{aligned}
\mathcal{E} & =\frac{\hbar \Omega K}{48}(\zeta(\alpha)+\zeta(-\alpha)-2) \\
& -\frac{\hbar \Omega}{8 \pi^{2} K}(\xi(\alpha) \zeta(\alpha)+\xi(-\alpha) \zeta(-\alpha)-2 \xi(0)) \\
\zeta(\alpha) & =\frac{1}{2}\left(\zeta_{u}(\alpha)+\zeta_{v}(\alpha)\right)
\end{aligned}
$$

The energy density is here directly expressed with respect to the static Casimir energy which is recovered when the cavity is motionless [2]. This result is due to the fact that the vacuum outside and inside the cavity is not the same but differs exactly by this amount of energy.

In order to obtain an appreciable value for the radiated energy if the cavity is moving at a small velocity, it is necessary to consider a high finesse cavity $\rho \ll 1$, keeping in mind that the finesse should be limited by the condition $\alpha_{\text {eff }}<1$. Using these assumptions equations $(29,30$ ) may be approximated as follows by expanding separately the common denominator and numerators

$$
\begin{aligned}
E & \approx \frac{\hbar \Omega}{6} \alpha^{2}+\frac{\hbar \Omega}{6}\left(1-\frac{1}{K^{2}}\right) \frac{\rho \alpha^{2}}{\rho^{2}-\alpha^{2}} \\
\mathcal{E} & \approx \frac{\hbar \Omega}{24}\left(K-\frac{1}{K}\right) \frac{\alpha^{2}}{\rho^{2}-\alpha^{2}} \\
\alpha & \leq \frac{\rho}{2} \ll 1
\end{aligned}
$$

The first term in the radiated energy is due to the field which is directly reflected by the two mirrors without entering the cavity. This term corresponds to the expression for motion-induced radiation from a single perfectly reflecting mirror. The second term has its origin in the field which has traversed the cavity and thus accumulated a much more important dephasing than the singly reflected field. Neglecting $\alpha^{2}$ in the denominator leads to the linear result presented in [9]. The linear approximation is found to be rigorously valid if the rapidity is much smaller than the cavity losses $(\alpha \ll \rho)$. Furthermore the present treatment allows us to calculate motion-induced radiation when the field dephasing becomes large due to accumulation on a large number of reflections. Equations (31) have a range of validity extending up to the threshold $\alpha_{\text {eff }}=1$.

The intracavity energy contains only the term corresponding to the field which has entered the cavity. Its expression can also be deduced from the radiated energy through a detailed balance argument, which goes as follows [9]: The energy inside the cavity can be obtained from the radiated energy by multiplying it by two factors $\Omega /(2 \pi)$ and $2 L /(4 \rho)$. The first factor is due to the fact that the radiated energy is the energy density integrated over one oscillation period. During one roundtrip of duration $2 L$ each photon has the probability $4 \rho$ - corresponding to the energy transmission coefficient of the two mirrors - to escape from the cavity. The present nonlinear evaluation of the intracavity energy gives indeed the same result as the balance argument in the limiting case of a high finesse cavity (cf. equations (31)). However, as equations (28,30) show the balance argument is not true for a cavity with arbitrary reflection and transmission coefficients. A remarkable consequence of equations (31) is that the non-linear calculation is necessary as soon as the number of photons inside the cavity becomes of the order of unity.

Interesting remarks can be made concerning the particular case $K=1$. Clearly equations (31) show that for a high finesse cavity no enhancement of photon production inside the cavity can be obtained when the mechanical excitation frequency equals the lowest cavity mode $(K=1)$ in accordance with results in ref. [5,6]. In this case the energy $E$ radiated by the cavity corresponds to the one emitted by a single mirror and the motional intracavity energy vanishes. However, in the general case of arbitrary cavity finesse motion-induced photons are also found for the $K=1$ mode (cf. equation (30)). The key point is that as far as classical light rays are concerned the mode $K=1$ behaves like all other modes [7]. However the field dephasing and thus motion-induced radiation is not 
only determined by the behavior of the light rays but also by the cavity which plays the part of a filtering function and suppresses photons at zero frequency. As a consequence of the coupling to radiation pressure photons are not emitted singly but in pairs. Thus motion-induced radiation is enhanced by the cavity if all photons are emitted into a cavity mode, the sum of their frequencies being equal to the mechanical oscillation frequency. In order to fulfill this condition when the cavity oscillates with the frequency of the lowest cavity mode photons have to be emitted at zero frequency. The cavity suppresses those photons the more efficiently the higher is the cavity finesse. Thus motion-induced photons for the $K=1$ mode can be found in the bad cavity limit but not in the high finesse limit.

Coming back to the general case, we emphasize that equations (31) remain valid up to the threshold $\rho=2 \alpha$, when the cavity finesse $\rho^{-1}$ is increased with the amplitude of motion kept constant. Below this value motioninduced radiation is amplified inside the cavity, but the cavity losses exceed the amplification gain. As expressions (31) are monotonous in $\rho$ their maximum values are thus reached at threshold. In this regime we then find a maximum of the radiated energy which depends linearly on $\rho$. Comparing this value to the maximum energy emitted by a single oscillating mirror shows a gain of the order of the cavity finesse by considering a cavity instead of a single mirror. The cavity is thus a much more favorable system to produce motion-induced radiation than a single mirror. Furthermore if one increases the cavity finesse above its threshold value the roundtrip amplification of the field due to the mirrors motion should exceed the cavity losses and the system should enter a regime of exponential amplification. Without further calculations we then expect the oscillating cavity to emit photon pulses of much higher intensity above threshold than below.

\section{FREQUENCY UP-CONVERSION}

We now turn to the calculation of the radiation spectrum where we will proceed as previously by first studying the case of a single moving mirror and afterwards the one of the oscillating cavity.

The scattering field equation (2) writes in Fourier space

$$
\begin{aligned}
\varphi_{\text {out }}[\nu] & =\sqrt{T} \varphi_{\text {in }}[\nu]+\sqrt{R} \int \mathrm{d} \bar{\nu} \frac{\Omega}{2 \pi} \psi_{\text {in }}[\bar{\nu}] \int \mathrm{d} u e^{i \Omega(\nu u+\bar{\nu} V(u))} \\
\nu & =\frac{\omega}{\Omega} \quad \bar{\nu}=\frac{\bar{\omega}}{\Omega}
\end{aligned}
$$

where we have introduced the reduced frequencies $\bar{\nu}$ and $\nu$ normalized with respect to the mechanical frequency $\Omega$. The field dephasing of the output field is determined by $V(u)$ and thus associated with the mirrors position $Q(u)$ which is easily calculated from (17)

$$
\Omega Q(u)=\Omega \frac{V(u)-u}{2}=\operatorname{arctg}\left(\frac{\beta \cos \Omega u}{1+\beta \sin \Omega u}\right)
$$

$e^{2 i \bar{\nu} Q(u)}$ is a periodic function and can thus be developed into a Fourier series with discrete coefficients $\gamma_{m}$

$$
e^{2 i \bar{\nu} \Omega Q(u)}=\sum_{m} \gamma_{m}[\bar{\nu}] e^{-i m \Omega u}
$$

which will determine the radiation spectrum. If the mirrors motion were sinusoidal these Fourier coefficients would be given by Bessel functions of different orders. We have now to evaluate these coefficients for a homographic trajectory (19).

To this aim, we first write the field dephasing (34) as

$$
e^{2 i \Omega \bar{\nu} Q(u)}=\left(\frac{1+i \beta e^{-i \Omega u}}{1-i \beta e^{i \Omega u}}\right)^{\bar{\nu}}
$$

The Fourier coefficients (34) may be rewritten in terms of an hypergeometric series

$$
\begin{aligned}
\gamma_{m}[\bar{\nu}] & =(-i)^{m+2} \frac{\bar{\nu}}{\pi} \sin (\pi \bar{\nu}) G_{m}(\bar{\nu}, \beta) \\
G_{m}(\nu, \beta) & =\beta^{m} \sum_{l \geq 0} \frac{\Gamma(\nu+l) \Gamma(m-\nu+l)}{\Gamma(m+1+l)} \frac{\beta^{2 l}}{l !}
\end{aligned}
$$

The radiation spectrum, that is the spectral density of the photon number per unit time and defined for positive frequencies, is then given by [9]

$$
\begin{aligned}
n_{\nu} & =R \sum_{m \geq \nu} \frac{\nu}{m-\nu}\left|\gamma_{m}[m-\nu]\right|^{2} \\
& =R \frac{\sin ^{2}(\pi \nu)}{\pi^{2}} \sum_{m>\nu} \nu(m-\nu)\left|G_{m}(\nu, \beta)\right|^{2}
\end{aligned}
$$

The total energy may be recovered as the integral of the spectrum as well as the integral of the energy density. The radiation spectrum vanishes for all values $\nu$ equal to a natural number, that is for all frequencies $\omega$ equal to a multiple of $\Omega$. The spectrum thus decomposes into a succession of arches, each limited by two successive multiples of the excitation frequency.

The upper expression corresponds to reflection upon a single moving mirror. In order to get the spectral distribution of radiation from the cavity, we now have to take into account the interferences between light rays having undergone different number of reflections inside the cavity. We proceed in the same manner as in the case of a single moving mirror by splitting the function $f_{p}$ into two parts. The first part is linear in the parameter $u$ while the second one $Q_{p}$ is induced by the motion and harmonic like the mirrors' motion

\footnotetext{
${ }^{1}$ The coefficient $G_{m}(\nu, \beta)$ is directly related to the hypergeometric function $F\left(\nu, m-\nu ; m+1 ; \beta^{2}\right)$ defined for instance in [11]. We have also used property 8.334 of the $\Gamma$-function in the same reference.
} 


$$
\begin{aligned}
f_{p}(u) & =u-p L+2 Q_{p}(u) \\
\Omega Q_{p}(u) & =\operatorname{arctg}\left(\frac{\beta_{p} \cos \Omega u}{1+\beta_{p} \sin \Omega u}\right) \\
\beta_{p} & =(-1)^{K} \operatorname{th}(p \alpha)
\end{aligned}
$$

The round-trip dephasing $2 L$ corresponds to the case of periodic orbits so that the function $Q_{p}$ vanishes at these points. The periodic function $e^{2 i \bar{\nu} Q_{p}}$ can be developed into a Fourier series with coefficients now depending on the number of round trips $p$. We proceed as previously to find the Fourier coefficients

$$
\begin{aligned}
\gamma_{m, p}[\bar{\nu}] & =\int_{0}^{\frac{2 \pi}{\Omega}} \frac{\Omega}{2 \pi} d u e^{i m \Omega u} e^{i K \pi \bar{\nu} p} e^{2 i \Omega \bar{\nu} Q_{p}(u)} \\
& =(-i)^{m+2} \bar{\nu} \frac{\sin (\pi \bar{\nu})}{\pi} e^{i K \pi \bar{\nu} p} G_{m}\left(\nu, \beta_{p}\right)
\end{aligned}
$$

and the radiation spectrum

$$
\begin{aligned}
n_{\nu}= & \frac{\sin ^{2}(\pi \nu)}{\pi^{2}} \sum_{m>\nu} \nu(m-\nu) \\
\times & \left\{\mid \sqrt{R_{1}} T_{2} \sum_{n \geq 0} r^{n} e^{-2 i \pi K \nu(n+1)} G_{m}\left(\nu, \beta_{2 n+1}\right)\right. \\
- & \left.\sqrt{R_{2}} G_{m}\left(\nu, \beta_{-1}\right)\right|^{2} \\
& \left.+T_{1} T_{2}\left|\sum_{n \geq 0} r^{n} e^{-2 i \pi K \nu n} G_{m}\left(\nu, \beta_{2 n}\right)\right|^{2}\right\}
\end{aligned}
$$

Let us mention that we recover the predictions of the linearized treatment for a motion with a small velocity by keeping only the lowest-order term $m=1$ in the hypergeometric series. The spectrum is then parabolic and found to be restricted to the frequency range corresponding to the first arch [9].

Figure 3 shows the radiation spectrum for an effective rapidity $\alpha_{\text {eff }}=0.9$ near the threshold of parametric oscillation. The spectrum shown here is plotted for a cavity oscillating globally at a frequency of $\Omega=3 \pi / L$. This means that the cavity performs three oscillations during one roundtrip of the field inside the cavity. The dashed line was obtained by putting formally $K=0$ in equation (40) which eliminates the phase factors responsible for the interferences. It may be interpreted as the spectrum of radiation emitted by a single oscillating mirror averaged over the effective velocity. Clearly photons can be created by higher-order harmonics of the motion as well as by the fundamental one as soon as the effective velocity becomes appreciable compared to the speed of light. As a striking consequence, photons are radiated at frequencies higher than the mechanical frequency $\Omega$. A process of frequency up-conversion thus exists in the opto-mechanical coupling between vacuum fluctuations and mechanical motion of scatterers.

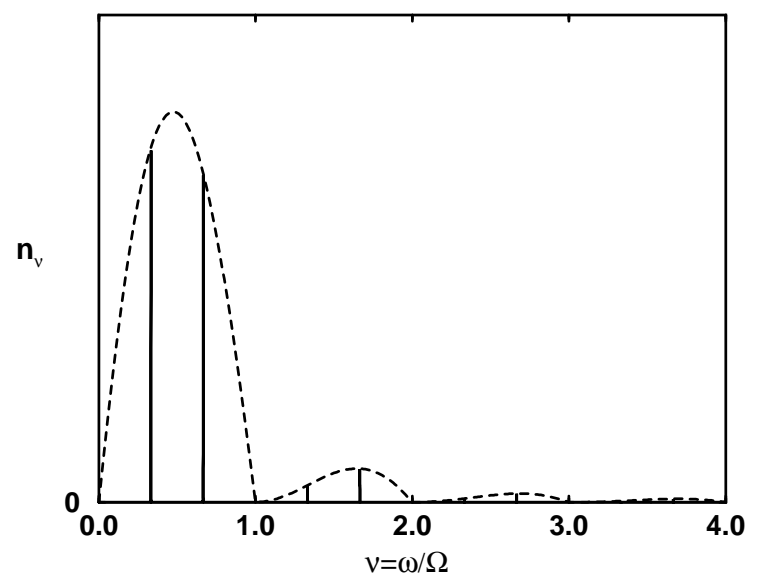

FIG. 3. Spectrum of the radiation emitted by the cavity for $\alpha_{\text {eff }}=0.9$ and a reflection coefficient of $r=0.99$. The top line of the frame corresponds to $\alpha_{\text {eff }}^{2} / 4$. The peaks correspond to cavity resonance frequencies. The spectrum is plotted for a cavity oscillating globally at a mechanical frequency $\Omega=3 \pi / L$. The dashed line constitutes the envelope of the spectrum. It was obtained by averaging the spectrum of a single mirror over the effective velocities corresponding to different number of roundtrips. Photons are created at frequencies higher than the mechanical oscillation frequency through frequency up-conversion in the opto-mechanical coupling between vacuum fluctuations and the mirrors motion. Furthermore the radiation spectrum vanishes for frequencies equal to a multiple integer of the mechanical excitation frequency.

A corresponding situation is found for a single oscillating mirror (dashed line) which is however not realistic as it would imply a mirror's mechanical velocity appreciable compared to the speed of light. The use of a cavity allows to reproduce the same spectral density within the bandwidth of the cavity resonance lines for realistic mirrors' velocities. A second striking feature is that no photons are emitted at frequencies equal to multiple integers of the excitation frequency $\Omega$ neither by a single oscillating mirror nor by a vibrating cavity.

When comparing the cavity radiation spectrum to expression (37) corresponding to a single mirror, a difference is the emergence of peaks typical of cavity resonances. In fact, the interferences between the pathes corresponding to different numbers $n$ of round-trips are essentially determined by the factors $r^{n} e^{-2 i n \pi K \nu}$ and $r^{n} e^{-2 i \pi K \nu(n+1)}$. The propagation dephasing after one round-trip is $e^{2 i \pi K \nu}$ where $K$ is the order of the mechanical frequency as compared with the fundamental resonance frequency of the cavity. It follows that the peaks are apparent at frequencies equal to an integer multiple of $K^{-1}$, as shown on Fig. 3 with $K=3$. Their shape is Lorentzian for a high finesse cavity. The width of each peak is given by the inverse of the cavity finesse. The number of peaks fitting into the interval $[0, \Omega]$ corresponds to the order $K$ of the excited cavity mode com- 
pared to the mechanical frequency.

\section{DISCUSSION}

In this paper we have presented a non-linear calculation of motion-induced radiation from a cavity taking fully into account the accumulation of dephasing through successive reflections of the field on partly transmitting mirrors. This approach confirms the main results of the linearized treatment which was previously used and makes it possible to specify its range of validity. Furthermore the non-linearity due to the accumulative field dephasing produces particular signatures of motion-induced radiation which cannot be calculated within the linear approximation.

In the experimentally relevant case where the mirrors move with a velocity small compared to the speed of light the emitted photon number from a single mirror moving in vacuum scales with its squared velocity. Compared to this situation motion-induced radiation from an oscillating cavity is enhanced by the cavity finesse. For high finesse cavities as they exist for instance in the microwaveregime this enhancement brings motion-induced radiation within reach of an experimental observation. This clearly proves the cavity to be a much more favorable system for the generation of motion-induced radiation.

In addition the present calculation shows that the linear approach is valid when the effective rapidity, given by the mirrors' physical velocity multiplied by the cavity finesse, is much smaller than 1 . We have given here expressions having a much larger range of validity.

In order to measure motion-induced radiation it is necessary to dispose of signatures which permit to distinguish vacuum radiation from spurious effects. The present calculation has allowed to identify two quantities showing signatures which could serve to this aim, the temporal variation of the radiated energy density and the spectral density of the emitted photon number.

We have studied the emitted energy density as a function of different effective rapidities. With increasing effective rapidity the energy starts to concentrate in pulses which are emitted periodically into vacuum by the cavity. These pulses become the higher and the sharper the more the effective rapidity approaches its threshold value. The energy density diverges when the single-reflection rapidity equals half of the cavity losses during one roundtrip $(\alpha=\rho / 2)$. The characteristic temporal variation which allows high energy densities in regularly spaced and narrow time windows might be exploited in an experimental observation.

The spectrum of motion-induced radiation shows several remarkable features. First photons may be radiated at frequencies higher than the mechanical frequency $\Omega$ in contrast to the prediction of the linear treatment. A process of frequency up-conversion thus takes place in the opto-mechanical coupling between vacuum fluc- tuations and mechanical motion of scatterers. Second the spectrum always vanishes for all multiple integers of the mechanical oscillation frequency. Due to the optomechanical resonance condition motion-induced radiation is furthermore only predicted at particular frequencies corresponding to fractions of the mechanical oscillation frequency. These signatures are different from pickup effects and could serve to identify motion-induced radiation.

So far we have discussed the behavior of the system in a regime where the cavity amplifies the dissipative effects of vacuum fluctuations. However, as a consequence of the divergence of the energy density there exists a threshold above which the system will show self-sustained oscillations in analogy with an optical parametric oscillator [10]. This regime is reached if the cavity finesse is increased above its threshold value. The amplification of motioninduced radiation should then exceed the cavity losses. It is to be expected that in this regime the cavity will emit photon pulses with much larger intensity than below threshold. If it were possible to reach this regime experimentally an observation of motion-induced radiation as well as of its characteristics could be achieved more easily. It might thus also be interesting to calculate the radiated energy and the spectrum above threshold. The question then arises which are the mechanisms limiting the amplification of radiation in this regime.

In conclusion, these results confirm the idea that it might be possible to show experimental evidence of the dissipative effects of motion in quantum vacuum.

Acknowledgements We thank M. Brune, M. Devoret, D. Estève, S. Haroche, J.-M. Raimond and C. Urbina for fruitful discussions.

[1] H.B.G. Casimir, Proc. K. Ned. Akad. Wet. 51793 (1948)

[2] S.A. Fulling and P.C.W. Davies, Proc. R. Soc. London A 348, 393 (1976)

[3] G.T. Moore, J. Math. Phys. 112679 (1970)

[4] V.V. Dodonov, A.B. Klimov and V.I. Man'Ko, Phys. Lett. A142 511 (1989)

[5] V.V. Dodonov, Phys. Lett. A207 126 (1995); see also references therein

[6] C.K. Law, Phys. Rev. Lett. 73, 1931 (1994)

[7] C.K. Cole and W.C. Schieve, Phys. Rev. 52, 4405 (1996)

[8] M.T. Jaekel and S. Reynaud, J. Phys. I France 1, 1395 (1991)

[9] A. Lambrecht, M.T. Jaekel, and S. Reynaud, Phys. Rev. Lett. 77, 615 (1996)

[10] S.Reynaud, A. Heidmann, E. Giacobino, and C. Fabre, Progress in Optics XXX, ed. E. Wolf, p.1-85 (1992)

[11] I.S. Gradshteyn, I.M. Ryzhik, Tables of Integrals, Series, and Products, 5th ed., ed. A. Jeffrey, Eqn. 9.100 